\title{
STOCHASTIC DIFFERENTIAL GAMES INVOLVING IMPULSE CONTROLS*
}

\author{
FENG ZHANG ${ }^{1}$
}

\begin{abstract}
A zero-sum stochastic differential game problem on infinite horizon with continuous and impulse controls is studied. We obtain the existence of the value of the game and characterize it as the unique viscosity solution of the associated system of quasi-variational inequalities. We also obtain a verification theorem which provides an optimal strategy of the game.
\end{abstract}

Mathematics Subject Classification. 91A15, 49N25, 49L20.

Received May 22, 2009. Revised August 5, 2009 and October 28, 2009.

Published online April 23, 2010.

\section{INTRODUCTION}

The study of differential games with Elliot-Kalton strategies in the viscosity solution framework was initiated by Evans and Souganidis [5]. Fleming and Souganidis [7] studied in a rigorous manner two-player zero-sum stochastic differential games and their work translated former results on differential games from the purely deterministic into the stochastic framework. Those interested in differential games are also referred to $[2,6]$ and the references therein.

Stochastic optimal control problems involving impulses were studied in many papers, especially for their wide applications in finance. See, for instance, Cadenillas and Zapatero [3], Korn [8], Øksendal and Sulem [9]. Yong [12] studied optimal control problems for systems governed by ordinary differential equations with continuous, switching and impulse controls. In Yong [13], deterministic differential game problems involving impulse controls were considered; one player takes continuous controls whereas the other uses impulse controls. Shaiju and Dharmatti [11] studied a zero-sum deterministic differential game problem, where the minimizer uses continuous, switching and impulse controls, while the maximizer takes continuous and switching controls. However, stochastic differential game problems involving impulse controls seem missing, to the author's knowledge.

In this paper, we study a zero-sum stochastic differential game problem, in which the maximizer uses continuous controls and the minimizer takes impulse controls. In Section 2, we formulate the stochastic differential game problem and establish the associated quasi-variational inequalities (QVI for short). In Section 3, by the dynamic programming principle we prove that the lower and upper value functions of the game satisfy the QVI in the viscosity solution sense. Then we establish the existence of the value by proving a uniqueness theorem for the QVI. Finally, we present a verification theorem which gives an optimal strategy of the game involving impulses in Section 4.

Keywords and phrases. Stochastic differential game, impulse control, quasi-variational inequalities, viscosity solution.

* This work was supported by the National Natural Science Foundation (10671112), the National Basic Research Program of China (973 Program, No. 2007CB814904), the Natural Science Foundation of Shandong Province (JQ200801) and the Doctoral Fund of Education Ministry of China (20060422018).

1 School of Mathematics, Shandong University, Jinan 250100, P.R. China. zhangfeng1104@gmail.com 


\section{Formulation of the Problem}

Let $B=\left(B_{t}\right)_{t \geq 0}$ be a $d$-dimensional standard Brownian motion, defined on a probability space $(\Omega, \mathscr{F}, \mathbb{P})$, equipped with the natural filtration $\left(\mathscr{F}_{t}\right)_{t \geq 0}$ satisfying the usual conditions. We are given $U$ a compact subset of $\mathbb{R}^{d}$ and $K$ a compact subset of $\mathbb{R}^{n}$. Let $\mathcal{U}$ denote the set of $U$-valued processes $u=\left(u_{r}\right)_{r>0}$ such that $u$ is $\left(\mathscr{F}_{r}\right)$-progressively measurable. We denote by $\mathcal{K}$ the set of processes $\eta(\cdot)=\sum_{i>1} \eta_{i} \mathbb{1}_{\left(\tau_{i}, \infty\right]}(\cdot)$ such that $\left(\tau_{i}\right) \subset[0, \infty],\left(\tau_{i}\right) \uparrow \infty$ a.s., $\tau_{i}$ is an $\mathscr{F}_{t}$-stopping time, and each $\eta_{i}: \Omega \rightarrow K$ is $\mathscr{F}_{\tau_{i}}$-measurable. $u \in \mathcal{U}$ is called the continuous control and $\eta \in \mathcal{K}$ is the impulse control.

Let us consider two functions $b: \mathbb{R}^{n} \times U \rightarrow \mathbb{R}^{n}$ and $\sigma: U \rightarrow \mathbb{R}^{n \times d}$ which satisfy:

(H1) There exist $\bar{\lambda}>0$ and $c>0$ such that for any $u \in U, x_{1}, x_{2} \in \mathbb{R}^{n}$,

$$
\left|b\left(x_{1}, u\right)-b\left(x_{2}, u\right)\right| \leq \bar{\lambda}\left|x_{1}-x_{2}\right|, \quad|b(0, u)|+|\sigma(u)| \leq c .
$$

Then, under (H1), the following stochastic differential equation (SDE):

$$
X^{x}(t)=x+\int_{0}^{t} b\left(X^{x}(s), u(s)\right) \mathrm{d} s+\int_{0}^{t} \sigma(u(s)) \mathrm{d} B_{s}+\xi(t)
$$

admits a unique solution $X^{x}(\cdot)$ for any $x \in \mathbb{R}^{n}$ and $(u, \xi) \in \mathcal{U} \times \mathcal{K}$. The cost functional $J^{x}: \mathcal{U} \times \mathcal{K} \rightarrow \mathbb{R}$ is given by

$$
J^{x}(u, \xi)=E\left\{\int_{0}^{\infty} \mathrm{e}^{-\lambda t} f\left(X^{x}(t), u(t)\right) \mathrm{d} t+\sum_{i \geq 1} \mathrm{e}^{-\lambda \tau_{i}} l\left(\xi_{i}\right) \mathbb{1}_{\left\{\tau_{i}<\infty\right\}}\right\}
$$

where $\lambda>0$ is a discount parameter, $f: \mathbb{R}^{n} \times U \rightarrow \mathbb{R}$ is the running cost function and $l: K \rightarrow \mathbb{R}$ is the impulse cost function. We assume that $\lambda, f$ and $l$ satisfy:

(H2) $\lambda>\bar{\lambda} ; f$ is continuous and bounded, and is uniformly Lipschitz in $x ; l$ is continuous and bounded. Moreover,

$$
\inf _{\xi \in K} l(\xi)=c>0 ; \quad l\left(\xi_{1}+\xi_{2}\right)<l\left(\xi_{1}\right)+l\left(\xi_{2}\right), \forall \xi_{1}, \xi_{2} \in K .
$$

Then it's easy to check that $J^{x}(u, \xi)$ is well defined for any $(u, \xi) \in \mathcal{U} \times \mathcal{K}$.

We now introduce the notion of nonanticipative strategies. The nonanticipative strategy set $\mathscr{A}$ for player- $\xi$ is the collection of all nonanticipative maps $\alpha$ from $\mathcal{U}$ to $\mathcal{K}$. The nonanticipative strategy set $\mathscr{B}$ for player- $u$ is the collection of all nonanticipative maps $\beta$ from $\mathcal{K}$ to $\mathcal{U}$. For the detailed definitions, one is referred to Definition 3.2 in [2]. Then for any $x \in \mathbb{R}^{n}, u \in \mathcal{U}$ and $\alpha \in \mathscr{A}$, we have $(u, \alpha(u)) \in \mathcal{U} \times \mathcal{K}$. Thus, SDE (2.1) admits a unique solution $X^{x}$ corresponding to $(u, \alpha(u))$, and the cost functional $J^{x}(u, \alpha(u))$ is well defined. Then we define the lower value function $V_{-}$and the upper value function $V_{+}$by

$$
\begin{aligned}
& V_{-}(x)=\inf _{\alpha \in \mathscr{A}} \sup _{u \in \mathcal{U}} J^{x}(u, \alpha(u)), \\
& V_{+}(x)=\sup _{\beta \in \mathscr{B}} \inf _{\xi \in \mathcal{K}} J^{x}(\beta(\xi), \xi) .
\end{aligned}
$$

If $V_{-}=V_{+}$, then we say that the game admits a value and $V:=V_{-}=V_{+}$is called the value function of the game. Furthermore, if there exists $\left(u^{*}, \xi^{*}\right) \in \mathcal{U} \times \mathcal{K}$ such that $V(x)=J^{x}\left(u^{*}, \xi^{*}\right)$, then we say that $\left(u^{*}, \xi^{*}\right)$ is an optimal strategy of the game.

For a continuous function $\phi: \mathbb{R}^{n} \rightarrow \mathbb{R}$, the minimum cost operator $N$ is defined by

$$
N[\phi](x)=\inf _{\xi \in K}\{\phi(x+\xi)+l(\xi)\} .
$$


Let us define the Hamiltonian $H: \mathbb{R}^{n} \times \mathbb{R} \times \mathbb{R}^{n} \times \mathcal{S}^{n} \rightarrow \mathbb{R}$ as follows:

$$
H(x, p, P, Q)=\sup _{u \in U}\left\{f(x, u)-\lambda p+b(x, u)^{*} P+\frac{1}{2} \operatorname{Tr}\left[\left(\sigma \sigma^{*}\right)(u) Q\right]\right\} .
$$

Then our stochastic differential game problem corresponds to the following QVI:

$$
\min \left\{H\left(x, V(x), D V(x), D^{2} V(x)\right), N[V](x)-V(x)\right\}=0, \quad x \in \mathbb{R}^{n},
$$

or equivalently,

$$
\left\{\begin{array}{l}
H\left(x, V(x), D V(x), D^{2} V(x)\right) \geq 0 \\
N[V](x) \geq V(x) \\
H\left(x, V(x), D V(x), D^{2} V(x)\right)(N[V](x)-V(x))=0
\end{array}\right.
$$

\section{Existence of the value}

In this section, we show that both $V_{-}$and $V_{+}$are viscosity solutions of QVI (2.3) and QVI (2.3) admits at most one viscosity solution. Thus $V_{-}$and $V_{+}$coincide. Hence, the value of our stochastic differential game exists.

We denote by $B U C\left(\mathbb{R}^{n}\right)$ the set of functions $\phi: \mathbb{R}^{n} \rightarrow \mathbb{R}$ such that $\phi$ is bounded and uniformly continuous. Let $C^{2}\left(\mathbb{R}^{n}\right)$ denote the set of $\mathbb{R}^{n} \rightarrow \mathbb{R}$ functions that are twice continuously differentiable with respect to their arguments.

Let us first recall the definition of a viscosity solution of QVI (2.3). The reader more interested in viscosity solutions is referred to Crandall et al. [4].

Definition 3.1. A function $V \in B U C\left(\mathbb{R}^{n}\right)$ is called:

(i) a viscosity subsolution of QVI (2.3) if for any $\varphi \in C^{2}\left(\mathbb{R}^{n}\right)$, whenever $V-\varphi$ attains a local maximum at $x \in \mathbb{R}^{n}$, it holds that

$$
\min \left\{H\left(x, \varphi(x), D \varphi(x), D^{2} \varphi(x)\right), N[V](x)-V(x)\right\} \geq 0
$$

(ii) a viscosity supersolution of QVI (2.3) if for any $\varphi \in C^{2}\left(\mathbb{R}^{n}\right)$, whenever $V-\varphi$ attains a local minimum at $x \in \mathbb{R}^{n}$, it holds that

$$
\min \left\{H\left(x, \varphi(x), D \varphi(x), D^{2} \varphi(x)\right), N[V](x)-V(x)\right\} \leq 0 ;
$$

(iii) a viscosity solution of QVI (2.3) if it's both a viscosity sub- and a supersolution of (2.3).

The main result of this section is:

Theorem 3.2. Assume (H1) and (H2). Then $V_{-}=V_{+}$is the unique viscosity solution of QVI (2.3) in $B U C\left(\mathbb{R}^{n}\right)$. Thus our stochastic differential game admits a value.

Before the proof of Theorem 3.2, some necessary preparations are needed.

Lemma 3.3. Assume (H1) and (H2). Then we have:

(i) $V_{-}, N\left[V_{-}\right] \in B U C\left(\mathbb{R}^{n}\right)$;

(ii) $V_{+}, N\left[V_{+}\right] \in B U C\left(\mathbb{R}^{n}\right)$.

Proof. We only prove (i). Let $\alpha_{0} \in \mathscr{A}$ be the strategy that for any $u \in \mathcal{U}, \alpha_{0}(u)$ makes no impulses. Then by the definition of $V_{-}$, we have

$$
V_{-}(x) \leq \sup _{u \in \mathcal{U}} J^{x}\left(u, \alpha_{0}(u)\right)=\sup _{u \in \mathcal{U}} E\left\{\int_{0}^{\infty} \mathrm{e}^{-\lambda t} f\left(X^{x}(t), u(t)\right) \mathrm{d} t\right\} \leq c .
$$

On the other hand, since the impulse cost $l$ is positive valued, for any $u \in \mathcal{U}$ and $\alpha \in \mathscr{A}$,

$$
J^{x}(u, \alpha(u)) \geq E\left\{\int_{0}^{\infty} \mathrm{e}^{-\lambda t} f\left(X^{x}(t), u(t)\right) \mathrm{d} t\right\} \geq-c .
$$

Thus, $V_{-}(x) \geq-c$. Hence we obtain $\left|V_{-}(x)\right| \leq c, \forall x \in \mathbb{R}^{n}$, and the boundedness of $V_{-}$is proved. 
Now let us prove the continuity of $V_{-}$. For any $x, y \in \mathbb{R}^{n}, u \in \mathcal{U}$ and $\alpha \in \mathscr{A}$, we have

$$
X^{x}(t)-X^{y}(t)=(x-y)+\int_{0}^{t}\left[b\left(X^{x}(s), u(s)\right)-b\left(X^{y}(s), u(s)\right)\right] \mathrm{d} s .
$$

Then by standard arguments it follows that $E\left|X^{x}(t)-X^{y}(t)\right| \leq \mathrm{e}^{\bar{\lambda} t}|x-y|$. Hence,

$$
\begin{aligned}
J^{x}(u, \alpha(u))-J^{y}(u, \alpha(u)) & =E\left\{\int_{0}^{\infty} \mathrm{e}^{-\lambda t}\left[f\left(X^{x}(t), u(t)\right)-f\left(X^{y}(t), u(t)\right)\right] \mathrm{d} t\right\} \\
& \leq c E\left\{\int_{0}^{\infty} \mathrm{e}^{-(\lambda-\bar{\lambda}) t}|x-y| \mathrm{d} t\right\}=\frac{c}{\lambda-\bar{\lambda}}|x-y|,
\end{aligned}
$$

which follows that

$$
\left|V_{-}(x)-V_{-}(y)\right| \leq \frac{c}{\lambda-\bar{\lambda}}|x-y|, \quad \forall x, y \in \mathbb{R}^{n} .
$$

Hence, $V_{-}$is uniformly continuous. We obtain that $V_{-} \in B U C\left(\mathbb{R}^{n}\right)$. And $N\left[V_{-}\right] \in B U C\left(\mathbb{R}^{n}\right)$ is an immediate consequence.

Subsequently, for $\eta(\cdot)=\sum_{i \geq 1} \eta_{i} \mathbb{1}_{\left(\tau_{i}, \infty\right]}(\cdot) \in \mathcal{K}$, we use the notation $(\eta)_{1, i}$ to denote $\tau_{i}$ and $(\eta)_{2, i}$ to denote $\eta_{i}$. We now present the dynamic programming principle (DPP) for our game problem.

Lemma 3.4. For any $x \in \mathbb{R}^{n}$ and $t>0$, we have:

(i)

$$
V_{-}(x)=\inf _{\alpha \in \mathscr{A}} \sup _{u \in \mathcal{U}} E\left\{\int_{0}^{t} \mathrm{e}^{-\lambda s} f\left(X^{x}(s), u(s)\right) \mathrm{d} s+\sum_{\alpha(u)_{1, i} \leq t} \mathrm{e}^{-\lambda \alpha(u)_{1, i}} l\left(\alpha(u)_{2, i}\right)+\mathrm{e}^{-\lambda t} V_{-}\left(X^{x}(t)\right)\right\},
$$

where $X^{x}(t)=X^{x}(t ; u, \alpha(u))$;

(ii)

$$
V_{+}(x)=\sup _{\beta \in \mathscr{B}} \inf _{\xi \in \mathcal{K}} E\left\{\int_{0}^{t} \mathrm{e}^{-\lambda s} f\left(X^{x}(s), \beta(\xi)(s)\right) \mathrm{d} s+\sum_{(\xi)_{1, i} \leq t} \mathrm{e}^{-\lambda(\xi)_{1, i}} l\left((\xi)_{2, i}\right)+\mathrm{e}^{-\lambda t} V_{+}\left(X^{x}(t)\right)\right\},
$$

where $X^{x}(t)=X^{x}(t ; \beta(\xi), \xi)$.

Proof. We only prove (i); the proof of (ii) is similar. Some proof ideas come from $[2,6,7]$.

Let $x$ be fixed, and let $W(x)$ be the right-hand side of (3.1). We fix $t, \varepsilon>0$. Then, there exists $\alpha^{1} \in \mathscr{A}$ such that for any $u(\cdot) \in \mathcal{U}$,

$$
W(x) \geq E\left\{\int_{0}^{t} \mathrm{e}^{-\lambda s} f\left(X^{x}(s), u(s)\right) \mathrm{d} s+\sum_{\alpha^{1}(u)_{1, i} \leq t} \mathrm{e}^{-\lambda \alpha^{1}(u)_{1, i}} l\left(\alpha^{1}(u)_{2, i}\right)+\mathrm{e}^{-\lambda t} V_{-}\left(X^{x}(t)\right)\right\}-\varepsilon
$$

where $X^{x}(t)=X^{x}\left(t ; u, \alpha^{1}(u)\right)$. By the definition of $V_{-}(x)$ and the properties of $V_{-}$and $J$, it's not difficult to deduce that there exists $\alpha^{2} \in \mathscr{A}$ such that

$$
E\left[V_{-}\left(X^{x}(t)\right)\right] \geq E\left[J^{X^{x}(t)}\left(u, \alpha^{2}(u)\right)\right]-3 \varepsilon \mathrm{e}^{-\lambda t}, \quad \forall u \in \mathcal{U}
$$


For the proof of issue (3.4), we follow the proof of Proposition 1.10 in [7]. Let $\left\{A_{i}, i=1,2, \ldots\right\}$ be a partition of $\mathbb{R}^{n}$, and choose $y_{i} \in A_{i}$. If the diameter of the $A_{i}$ 's is sufficiently small, then for $y \in A_{i}$, the uniform continuity of $J$ and $V_{-}$yields

$$
J^{y_{i}}(u, \alpha(u)) \geq J^{y}(u, \alpha(u))-\varepsilon \mathrm{e}^{-\lambda t}, \forall u \in \mathcal{U}, \alpha \in \mathscr{A},
$$

and

$$
V_{-}(y) \geq V_{-}\left(y_{i}\right)-\varepsilon \mathrm{e}^{-\lambda t} .
$$

By the definition of $V_{-}$, there exists $\alpha^{y_{i}} \in \mathscr{A}$ such that

$$
V_{-}\left(y_{i}\right) \geq J^{y_{i}}\left(u, \alpha^{y_{i}}(u)\right)-\varepsilon \mathrm{e}^{-\lambda t}, \forall u \in \mathcal{U} .
$$

Thus, for $y \in A_{i}$, we have

$$
V_{-}(y) \geq J^{y}\left(u, \alpha^{y_{i}}(u)\right)-3 \varepsilon \mathrm{e}^{-\lambda t}, \forall u \in \mathcal{U}
$$

It follows that

$$
\begin{aligned}
E\left[V_{-}\left(X^{x}(t)\right)\right] & =E\left[\sum_{i=1}^{\infty} \mathbb{1}_{A_{i}}\left(X^{x}(t)\right) V_{-}\left(X^{x}(t)\right)\right] \\
& \geq E\left[\sum_{i=1}^{\infty} \mathbb{1}_{A_{i}}\left(X^{x}(t)\right) J^{X^{x}(t)}\left(u, \alpha^{y_{i}}(u)\right)\right]-3 \varepsilon \mathrm{e}^{-\lambda t}
\end{aligned}
$$

Now we define a mapping $\alpha^{2}$ by

$$
\alpha^{2}(u)(s)=\sum_{i=1}^{\infty} \mathbb{1}_{A_{i}}\left(X^{x}(t)\right) \alpha^{y_{i}}(u)(s), s>t .
$$

Then $\alpha^{2}$ is nonanticipative and the relation (3.4) follows immediately. Consequently, by (3.3) and (3.4), we get

$$
\begin{aligned}
W(x) \geq & E\left\{\int_{0}^{t} \mathrm{e}^{-\lambda s} f\left(X^{x}(s), u(s)\right) \mathrm{d} s\right. \\
& \left.+\sum_{\alpha^{1}(u)_{1, i} \leq t} \mathrm{e}^{-\lambda \alpha^{1}(u)_{1, i}} l\left(\alpha^{1}(u)_{2, i}\right)+\mathrm{e}^{-\lambda t} J^{X^{x}(t)}\left(u, \alpha^{2}(u)\right)\right\}-4 \varepsilon .
\end{aligned}
$$

Now we define a strategy $\delta$ by

$$
\delta(u(\cdot))(s)= \begin{cases}\alpha^{1}(u(\cdot))(s), & s \leq t \\ \alpha^{2}(u(\cdot+t))(s-t), & s>t\end{cases}
$$

It's obvious that $\delta$ is a nonanticipative map since $\alpha^{1}$ and $\alpha^{2}$ are both so. Then, by change of variables, it follows from (3.5) that

$$
W(x) \geq E\left\{\int_{0}^{\infty} \mathrm{e}^{-\lambda t} f\left(X^{x}(t), u(t)\right) \mathrm{d} t+\sum_{i \geq 1} \mathrm{e}^{-\lambda \delta(u)_{1, i}} l\left(\delta(u)_{2, i}\right)\right\}-4 \varepsilon,
$$

where $X^{x}(t)=X^{x}(t ; u, \delta(u))$. That is, $W(x) \geq J^{x}(u, \delta(u))-4 \varepsilon$. By the arbitrariness of $u$, we obtain $W(x) \geq$ $V_{-}(x)-4 \varepsilon$, which follows immediately that $W(x) \geq V_{-}(x)$. 
We now prove the inequality $W(x) \leq V_{-}(x)$. For fixed $\alpha \in \mathscr{A}$ and $\varepsilon>0$, there exists $u^{1} \in \mathcal{U}$ such that

$$
W(x) \leq E\left\{\int_{0}^{t} \mathrm{e}^{-\lambda s} f\left(X^{x}(s), u^{1}(s)\right) \mathrm{d} s+\sum_{\alpha\left(u^{1}\right)_{1, i} \leq t} \mathrm{e}^{-\lambda \alpha\left(u^{1}\right)_{1, i}} l\left(\alpha\left(u^{1}\right)_{2, i}\right)+\mathrm{e}^{-\lambda t} V_{-}\left(X^{x}(t)\right)\right\}+\varepsilon,
$$

where $X^{x}(t)=X^{x}\left(t ; u^{1}, \alpha\left(u^{1}\right)\right)$. By the definition of $V_{-}(x)$, with the similar arguments that lead to (3.4), we get that there exists $u^{2} \in \mathcal{U}$ such that

$$
E\left[V_{-}\left(X^{x}(t)\right)\right] \leq E\left[J^{X^{x}(t)}\left(u^{2}, \alpha\left(u^{2}\right)\right)\right]+\varepsilon \mathrm{e}^{-\lambda t} .
$$

Now we define $\tilde{u} \in \mathcal{U}$ as follows:

$$
\tilde{u}(s)= \begin{cases}u^{1}(s), & s \leq t \\ u^{2}(s-t), & s>t .\end{cases}
$$

Then by (3.6), (3.7) and change of variables, we obtain

$$
W(x) \leq E\left\{\int_{0}^{\infty} \mathrm{e}^{-\lambda t} f\left(X^{x}(t), \tilde{u}(t)\right) \mathrm{d} t+\sum_{i \geq 1} \mathrm{e}^{-\lambda \alpha(\tilde{u})_{1, i}} l\left(\alpha(\tilde{u})_{2, i}\right) \mathbb{1}_{\left\{\alpha(\tilde{u})_{1, i}<\infty\right\}}\right\}+2 \varepsilon,
$$

where $X^{x}(t)=X^{x}(t ; \tilde{u}, \alpha(\tilde{u}))$. That is, $W(x) \leq J^{x}(\tilde{u}, \alpha(\tilde{u}))+2 \varepsilon$. Finally, the arbitrariness of $\alpha$ and $\varepsilon$ follows that $W(x) \leq V_{-}(x)$.

As a consequence of the DPP, we have:

Lemma 3.5. The lower and upper value functions $V_{\mp}$ satisfy the following:

(i) $V_{\mp}(x) \leq N\left[V_{\mp}\right](x), \forall x \in \mathbb{R}^{n}$.

(ii) Let $x \in \mathbb{R}^{n}$ be such that a strict inequality holds in (i) (for $V_{-}$or $V_{+}$). Then, (correspondingly) there exists $t_{0}>0$ such that for any $u \in \mathcal{U}$ and $t \leq t_{0}$,

$$
V_{\mp}(x)+t^{2}>E\left\{\int_{0}^{t} \mathrm{e}^{-\lambda s} f\left(X^{x}(s), u(s)\right) \mathrm{d} s+\mathrm{e}^{-\lambda t} V_{\mp}\left(X^{x}(t)\right)\right\},
$$

where $X^{x}(\cdot)$ satisfies

$$
X^{x}(t)=x+\int_{0}^{t} b\left(X^{x}(s), u(s)\right) \mathrm{d} s+\int_{0}^{t} \sigma(u(s)) \mathrm{d} B_{s} .
$$

Proof. We only prove the assertions for $V_{-}$. We obtain (i) by letting $t \downarrow 0$ in (3.1). By Lemma 3.4-(i), for any $t>0$, there exists $\alpha^{t} \in \mathscr{A}$ such that for any $u \in \mathcal{U}$,

$$
V_{-}(x)+t^{2}>E\left\{\int_{0}^{t} \mathrm{e}^{-\lambda s} f\left(X^{x}(s), u(s)\right) \mathrm{d} s+\sum_{\alpha^{t}(u)_{1, i} \leq t} \mathrm{e}^{-\lambda \alpha^{t}(u)_{1, i}} l\left(\alpha^{t}(u)_{2, i}\right)+\mathrm{e}^{-\lambda t} V_{-}\left(X^{x}(t)\right)\right\},
$$

where $X^{x}(t)=X^{x}\left(t ; u, \alpha^{t}(u)\right)$. To get (ii) for $V_{-}$, it's enough to show that, for some $t_{0}>0, \theta^{t}:=\alpha^{t}(u)_{1,1} \geq t$ for any $0 \leq t \leq t_{0}$. If this is not true, then (without loss of generality) there would be a sequence $t_{n} \downarrow 0$ such that $\theta^{t_{n}}<t_{n}$. This implies that

$$
V_{-}(x)+\left(\theta^{t_{n}}\right)^{2}>E\left\{\int_{0}^{\theta^{t_{n}}} \mathrm{e}^{-\lambda s} f\left(X^{x}(s), u(s)\right) \mathrm{d} s+\mathrm{e}^{-\lambda \theta^{t_{n}}} l\left(\alpha^{\theta^{t_{n}}}(u)_{2,1}\right)+\mathrm{e}^{-\lambda \theta^{t_{n}}} V_{-}\left(X^{x}\left(\theta^{t_{n}}\right)\right)\right\} .
$$

Then by letting $n \rightarrow \infty$ we obtain that $V_{-}(x) \geq N\left[V_{-}\right](x)$, which contradicts the hypothesis that a strict inequality holds in (i). The proof is concluded. 
We are now ready to prove:

Proposition 3.6. Under (H1) and (H2), $V_{-}$and $V_{+}$are viscosity solutions of $Q V I(2.3)$.

Proof. We only prove that $V_{-}$is a viscosity solution of QVI (2.3). Let us show in a first step that $V_{-}$is a viscosity subsolution. For this, we suppose that $\varphi \in C^{2}\left(\mathbb{R}^{n}\right)$ and $x \in \mathbb{R}^{n}$ are such that $V_{-}-\varphi$ attains a local maximum at $x$. Without loss of generality, we assume that $V_{-}(x)=\varphi(x)$. From Lemma 3.5, we have $N\left[V_{-}\right](x)-V_{-}(x) \geq 0$, so it suffices to prove that $H\left(x, \varphi(x), D \varphi(x), D^{2} \varphi(x)\right) \geq 0$. To this end, let $\alpha_{0} \in \mathscr{A}$ be the strategy that for any $u \in \mathcal{U}, \alpha_{0}(u)$ makes no impulses. Then by Lemma 3.4, for any $\varepsilon>0$, there exists $u^{\varepsilon}(\cdot) \in \mathcal{U}$ such that

$$
\begin{aligned}
\varphi(x) & \leq \sup _{u \in \mathcal{U}} J^{x}\left(u, \alpha_{0}(u)\right) \\
& \leq E\left\{\int_{0}^{t} \mathrm{e}^{-\lambda s} f\left(X^{x}(s), u^{\varepsilon}(s)\right) \mathrm{d} s+\mathrm{e}^{-\lambda t} V_{-}\left(X^{x}(t)\right)\right\}+\varepsilon t \\
& \leq E\left\{\int_{0}^{t} \mathrm{e}^{-\lambda s} f\left(X^{x}(s), u^{\varepsilon}(s)\right) \mathrm{d} s+\mathrm{e}^{-\lambda t} \varphi\left(X^{x}(t)\right)\right\}+\varepsilon t
\end{aligned}
$$

where $X^{x}(\cdot)$ satisfies

$$
X^{x}(t)=x+\int_{0}^{t} b\left(X^{x}(s), u^{\varepsilon}(s)\right) \mathrm{d} s+\int_{0}^{t} \sigma\left(u^{\varepsilon}(s)\right) \mathrm{d} B_{s},
$$

for $t>0$ small enough. By Itô's formula applied to $\mathrm{e}^{-\lambda t} \varphi\left(X^{x}(t)\right)$, we obtain

$$
\begin{aligned}
0 \leq & \varepsilon t-\lambda E \int_{0}^{t} \mathrm{e}^{-\lambda s} \varphi\left(X^{x}(s)\right) \mathrm{d} s+E \int_{0}^{t} \mathrm{e}^{-\lambda s} f\left(X^{x}(s), u^{\varepsilon}(s)\right) \mathrm{d} s \\
& +E \int_{0}^{t} \mathrm{e}^{-\lambda s}\left\{b^{*}\left(X^{x}(s), u^{\varepsilon}(s)\right) D \varphi\left(X^{x}(s)\right)+\frac{1}{2} \operatorname{Tr}\left[\left(\sigma \sigma^{*}\right)\left(u^{\varepsilon}(s)\right) D^{2} \varphi\left(X^{x}(s)\right)\right]\right\} \mathrm{d} s .
\end{aligned}
$$

Dividing $t$ and letting $t \downarrow 0$, we get that for some $u^{\varepsilon} \in U$,

$$
0 \leq f\left(x, u^{\varepsilon}\right)-\lambda \varphi(x)+b^{*}\left(x, u^{\varepsilon}\right) D \varphi(x)+\frac{1}{2} \operatorname{Tr}\left[\left(\sigma \sigma^{*}\right)\left(u^{\varepsilon}\right) D^{2} \varphi(x)\right]+\varepsilon,
$$

which follows $H\left(x, \varphi(x), D \varphi(x), D^{2} \varphi(x)\right)+\varepsilon \geq 0$. From the arbitrariness of $\varepsilon$, we obtain $H(x, \varphi(x), D \varphi(x)$, $\left.D^{2} \varphi(x)\right) \geq 0$.

We now proceed to prove that $V_{-}$is a viscosity supersolution. Let $\varphi \in C^{2}\left(\mathbb{R}^{n}\right)$ and $x \in \mathbb{R}^{n}$ be such that $V_{-}-\varphi$ attains a local minimum at $x$. Without loss of generality, we assume again that $V_{-}(x)=\varphi(x)$. If $N\left[V_{-}\right](x)-V_{-}(x)=0$, then there's nothing to prove. If $N\left[V_{-}\right](x)-V_{-}(x)>0$, we need to prove that $H\left(x, \varphi(x), D \varphi(x), D^{2} \varphi(x)\right) \leq 0$. By Lemma 3.5, there exists $t_{0}>0$ such that for any $u \in U$ and $t \leq t_{0}$,

$$
V_{-}(x)+t^{2}>E\left\{\int_{0}^{t} \mathrm{e}^{-\lambda s} f\left(X^{x}(s), u\right) \mathrm{d} s+\mathrm{e}^{-\lambda t} V_{-}\left(X^{x}(t)\right)\right\},
$$

where $X^{x}(\cdot)$ satisfies

$$
X^{x}(t)=x+\int_{0}^{t} b\left(X^{x}(s), u\right) \mathrm{d} s+\int_{0}^{t} \sigma(u) \mathrm{d} B_{s} .
$$


By Itô's formula, we obtain that

$$
\begin{aligned}
t^{2}> & -\lambda E \int_{0}^{t} \mathrm{e}^{-\lambda s} \varphi\left(X^{x}(s)\right) \mathrm{d} s+E \int_{0}^{t} \mathrm{e}^{-\lambda s} f\left(X^{x}(s), u\right) \mathrm{d} s \\
& +E \int_{0}^{t} \mathrm{e}^{-\lambda s}\left\{b^{*}\left(X^{x}(s), u\right) D \varphi\left(X^{x}(s)\right)+\frac{1}{2} \operatorname{Tr}\left[\left(\sigma \sigma^{*}\right)(u) D^{2} \varphi\left(X^{x}(s)\right)\right]\right\} \mathrm{d} s .
\end{aligned}
$$

By dividing $t$ and letting $t \downarrow 0$, we get

$$
0 \geq f(x, u)-\lambda \varphi(x)+b^{*}(x, u) D \varphi(x)+\frac{1}{2} \operatorname{Tr}\left[\left(\sigma \sigma^{*}\right)(u) D^{2} \varphi(x)\right], \quad \forall u \in U,
$$

which follows that $H\left(x, \varphi(x), D \varphi(x), D^{2} \varphi(x)\right) \leq 0$. The proof is complete.

We will show that QVI (2.3) admits at most one viscosity solution. A necessary lemma is given in the following. For the proof, one is referred to Lemma 4.4 in [12].

Lemma 3.7. Suppose $V$ is a uniformly continuous function. Let $y_{0} \in \mathbb{R}^{n}$ and $\xi_{0} \in K$ be such that

$$
V\left(y_{0}\right)=N[V]\left(y_{0}\right)=V\left(y_{0}+\xi_{0}\right)+l\left(\xi_{0}\right) .
$$

Then there exists $\delta>0$ which depends on $V$, such that

$$
V(y)<N[V](y), \quad \forall\left|y-\left(y_{0}+\xi_{0}\right)\right| \leq \delta .
$$

Now let us give the following:

Proposition 3.8. Assume $(\mathrm{H} 1)$ and $(\mathrm{H} 2)$. Let $v$ and $\omega$ be viscosity solutions of $Q V I(2.3)$ in $B U C\left(\mathbb{R}^{n}\right)$. Then $v=\omega$.

Proof. We only prove $v \leq \omega$. The inequality of the opposite direction can be proved similarly. To this end, we assume that $\sup _{x \in \mathbb{R}^{n}}(v(x)-\omega(x))>0$. Then we can find $\bar{x} \in \mathbb{R}^{n}$ and $\delta>0$ such that

$$
\sup _{x \in \mathcal{O}(\bar{x})}(v(x)-\omega(x))>0
$$

and

$$
N[\omega](x)>\omega(x), \quad \forall x \in \mathcal{O}(\bar{x}),
$$

where $\mathcal{O}(\bar{x})=\left\{x \in \mathbb{R}^{n}:|x-\bar{x}|<\delta\right\}$. In fact, there exists $y_{0} \in \mathbb{R}^{n}$ such that $v\left(y_{0}\right)-\omega\left(y_{0}\right)>0$. If $N[\omega]\left(y_{0}\right)>$ $\omega\left(y_{0}\right)$, then considering the uniform continuity of $v, \omega$ and $N[\omega]$, we obtain (3.8) and (3.9) by taking $\bar{x}=y_{0}$. Suppose it's not the case. Then let us assume that for some $\xi_{0} \in K, \omega\left(y_{0}\right)=N[\omega]\left(y_{0}\right)=\omega\left(y_{0}+\xi_{0}\right)+l\left(\xi_{0}\right)$. We choose $\bar{x}=y_{0}+\xi_{0}$. Then by Lemma 3.7, there exists $\delta>0$ such that $N[\omega](x)>\omega(x), \forall x \in \mathcal{O}(\bar{x})$. On the other hand, it's easy to check that $v(\bar{x})-\omega(\bar{x})=v(\bar{x})+l\left(\xi_{0}\right)-\omega\left(y_{0}\right) \geq v\left(y_{0}\right)-\omega\left(y_{0}\right)>0$. Hence, we obtain (3.8) and (3.9). In the sequel, we assume, without loss of generality, that

$$
v(x) \leq \omega(x), \quad \forall|x-\bar{x}|=\delta .
$$

Now let us define the Hamiltonian $\mathcal{H}: \mathbb{R}^{n} \times \mathbb{R} \times \mathbb{R}^{n} \times \mathcal{S}^{n} \rightarrow \mathbb{R}$ as follows:

$$
\mathcal{H}(x, p, P, Q)=\inf _{u \in U}\left\{-f(x, u)+\lambda p-b(x, u)^{*} P-\frac{1}{2} \operatorname{Tr}\left[\left(\sigma \sigma^{*}\right)(u) Q\right]\right\} .
$$


Then it's obvious that $\mathcal{H}=-H$. By Definition 3.1 and Proposition 3.6, together with (3.9), it's easy to check that, for any $\varphi \in C^{2}\left(\mathbb{R}^{n}\right)$, if $v-\varphi$ attains a local maximum at $x \in \mathcal{O}(\bar{x})$, then $\mathcal{H}\left(x, \varphi(x), D \varphi(x), D^{2} \varphi(x)\right) \leq 0$, and if $\omega-\varphi$ attains a local minimum at $x \in \mathcal{O}(\bar{x})$, then $\mathcal{H}\left(x, \varphi(x), D \varphi(x), D^{2} \varphi(x)\right) \geq 0$. Hence, in $\mathcal{O}(\bar{x}), v$ and $\omega$ are, respectively, viscosity subsolution and supersolution of the following equation:

$$
\mathcal{H}\left(x, V(x), D V(x), D^{2} V(x)\right)=0 .
$$

It's easy to check that the function $\mathcal{H}$ is proper in the sense that

$$
\mathcal{H}\left(x, p_{1}, P, Q_{1}\right) \leq \mathcal{H}\left(x, p_{2}, P, Q_{2}\right), \quad \text { if } p_{1} \leq p_{2} \text { and } Q_{1} \geq Q_{2} .
$$

And for any $p_{1} \geq p_{2}$ and $(x, P, Q) \in \mathbb{R}^{n} \times \mathbb{R}^{n} \times \mathcal{S}^{n}$,

$$
\mathcal{H}\left(x, p_{1}, P, Q\right)-\mathcal{H}\left(x, p_{2}, P, Q\right)=\lambda\left(p_{1}-p_{2}\right) .
$$

Now let us assume that $Q_{1}, Q_{2} \in \mathcal{S}^{n}, \alpha>0$ satisfy the following inequality:

$$
\left(\begin{array}{cc}
Q_{2} & 0 \\
0 & -Q_{1}
\end{array}\right) \leq \alpha\left(\begin{array}{cc}
I & -I \\
-I & I
\end{array}\right)
$$

Then it's easy to check that $Q_{2}-Q_{1} \leq 0$, and thus $\operatorname{Tr}\left[\left(\sigma \sigma^{*}\right)(u)\left(Q_{2}-Q_{1}\right)\right] \leq 0$. From the Lipschitz properties of $b$ and $f$, it follows that

$$
\mathcal{H}\left(y, p, \alpha(x-y), Q_{1}\right)-\mathcal{H}\left(x, p, \alpha(x-y), Q_{2}\right) \leq c\left(\alpha|x-y|^{2}+|x-y|\right) .
$$

Then by (3.10) and (3.12), we conclude from Theorem 3.3 in [4] that

$$
\sup _{x \in \overline{\mathcal{O}}(\bar{x})}(v(x)-\omega(x)) \leq 0
$$

which contradicts (3.8). Hence, $\sup _{x \in \mathbb{R}^{n}}(v(x)-\omega(x)) \leq 0$ and the proof is concluded.

Proof of Theorem 3.2. Theorem 3.2 is easily obtained by Lemma 3.3, Proposition 3.6 and Proposition 3.8.

\section{A VERIFICATION THEOREM}

In $[1,3,8]$, verification theorems were considered for stochastic optimal control problems involving impulses, which provide the optimal controls. In this section, we present a verification theorem which gives an optimal strategy of our zero-sum stochastic differential game.

We suppose that a classical solution of QVI (2.3) exists, denoted by $v$. Then $v$ separates the space $\mathbb{R}^{n}$ into two disjoint regions: a continuation region

$$
\mathcal{C}:=\left\{x \in \mathbb{R}^{n}: v(x)<N[v](x) \text { and } H\left(x, v(x), D v(x), D^{2} v(x)\right)=0\right\}
$$

and an intervention region

$$
\Sigma:=\left\{x \in \mathbb{R}^{n}: v(x)=N[v](x) \text { and } H\left(x, v(x), D v(x), D^{2} v(x)\right) \geq 0\right\} .
$$

In the sequel, we assume that for any $(u(\cdot), \xi(\cdot)) \in \mathcal{U} \times \mathcal{K}$,

$$
\lim _{t \rightarrow \infty} E\left[\mathrm{e}^{-\lambda t} v\left(X_{t+}\right)\right]=0,
$$


and

$$
E \int_{0}^{\infty}\left|\mathrm{e}^{-\lambda s} v^{\prime}\left(X_{s}\right) \sigma\left(u_{s}\right)\right|^{2} \mathrm{~d} s<\infty
$$

where $X(\cdot)$ is the solution of $\operatorname{SDE}(2.1)$ corresponding to $(u(\cdot), \xi(\cdot))$. We define, for any $u \in U$, the operator $\mathcal{L}^{u}$ by

$$
\mathcal{L}^{u} \varphi(x)=-\lambda \varphi(x)+b^{*}(x, u) D \varphi(x)+\frac{1}{2} \operatorname{Tr}\left[\left(\sigma \sigma^{*}\right)(u) D^{2} \varphi(x)\right], \quad x \in \mathbb{R}^{n}, \varphi \in C^{2}\left(\mathbb{R}^{n}\right) .
$$

Firstly we give the following:

Definition 4.1. The following mixed continuous-impulse stochastic control

$$
\left(u^{*}(\cdot), \xi^{*}(\cdot)\right)=\left(u^{*}(\cdot), \sum_{i \geq 1} \xi_{i}^{*} \mathbb{1}_{\left(\tau_{i}^{*}, \infty\right]}(\cdot)\right)
$$

is called the QVI-control associated with $v$ (if it exists):

$$
\begin{aligned}
& \mathbb{P}\left\{\forall\left(t, X_{t}\right) \in \mathbb{R}^{n} \times \mathcal{C}: u^{*}(t) \in \arg \max _{u \in U}\left\{\mathcal{L}^{u} v\left(X_{t}\right)+f\left(X_{t}, u\right)\right\}\right\}=1 \\
& \tau_{0}^{*}=0, \quad \xi_{0}^{*}=0 \\
& \tau_{1}^{*}=\inf \left\{t>0: v\left(X_{t}\right)=N[v]\left(X_{t}\right)\right\} \\
& \xi_{1}^{*}=\arg \inf \left\{v\left(X\left(\tau_{1}^{*}\right)+\xi\right)+l(\xi), \xi \in K\right\}
\end{aligned}
$$

and, for every $n \geq 2$,

$$
\begin{aligned}
& \tau_{n}^{*}=\inf \left\{t>\tau_{n-1}^{*}: v\left(X_{t}\right)=N[v]\left(X_{t}\right)\right\}, \\
& \xi_{n}^{*}=\arg \inf \left\{v\left(X\left(\tau_{n}^{*}\right)+\xi\right)+l(\xi), \xi \in K\right\} .
\end{aligned}
$$

Here, $X$ is the trajectory corresponding to $\left(u^{*}(\cdot), \xi^{*}(\cdot)\right)$.

We are now ready to present the verification theorem for our stochastic differential game problem involving impulses.

Theorem 4.2. Assume (H1) and (H2). Let $v \in B U C\left(\mathbb{R}^{n}\right)$ be a classical solution of $Q V I$ (2.3). If the QVIcontrol $\left(u^{*}(\cdot), \xi^{*}(\cdot)\right)$ associated with $v$ is admissible, then $v$ is the value function of our stochastic differential game. Thus, $\left(u^{*}(\cdot), \xi^{*}(\cdot)\right)$ is an optimal strategy of the game.

Proof. By Theorem 3.2 we know that $V:=V_{-}=V_{+}$is the unique viscosity solution of QVI (2.3) in $B U C\left(\mathbb{R}^{n}\right)$. Hence, we obtain

$$
v(x)=\inf _{\alpha \in \mathscr{A}} \sup _{u \in \mathcal{U}} J^{x}(u, \alpha(u))=\sup _{\beta \in \mathscr{B}} \inf _{\xi \in \mathcal{K}} J^{x}(\beta(\xi), \xi) .
$$

We now only need to show that $v(x)=J^{x}\left(u^{*}(\cdot), \xi^{*}(\cdot)\right)$. By an appropriate version of Itô's formula (see, e.g., Sect. IV.45 of [10]), we obtain for any $t>0$ and $n \geq 1$,

$$
\begin{aligned}
\mathrm{e}^{-\lambda\left(t \wedge \tau_{n}^{*}\right)} v\left(X_{\left(t \wedge \tau_{n}^{*}\right)+}\right)-v(x)= & \sum_{i=1}^{n}\left\{\int_{t \wedge \tau_{i-1}^{*}}^{t \wedge \tau_{i}^{*}} \mathrm{e}^{-\lambda s} \mathcal{L}^{u_{s}^{*}}\left(X_{s}\right) \mathrm{d} s+\int_{t \wedge \tau_{i-1}^{*}}^{t \wedge \tau_{i}^{*}} \mathrm{e}^{-\lambda s} v^{\prime}\left(X_{s}\right) \sigma\left(u_{s}^{*}\right) \mathrm{d} B_{s}\right\} \\
& +\sum_{i=1}^{n} \mathbb{1}_{\left\{\tau_{i}^{*} \leq t\right\}} \mathrm{e}^{-\lambda \tau_{i}^{*}}\left\{v\left(X_{\tau_{i}^{*}+}\right)-v\left(X_{\tau_{i}^{*}}\right)\right\} .
\end{aligned}
$$


From Definition 4.1, we have

$$
\begin{aligned}
\mathrm{e}^{-\lambda\left(t \wedge \tau_{n}^{*}\right)} v\left(X_{\left(t \wedge \tau_{n}^{*}\right)+}\right)-v(x)= & \sum_{i=1}^{n}\left\{-\int_{t \wedge \tau_{i-1}^{*}}^{t \wedge \tau_{i}^{*}} \mathrm{e}^{-\lambda s} f\left(X_{s}, u_{s}^{*}\right) \mathrm{d} s+\int_{t \wedge \tau_{i-1}^{*}}^{t \wedge \tau_{i}^{*}} \mathrm{e}^{-\lambda s} v^{\prime}\left(X_{s}\right) \sigma\left(u_{s}^{*}\right) \mathrm{d} B_{s}\right\} \\
& -\sum_{i=1}^{n} \mathbb{1}_{\left\{\tau_{i}^{*} \leq t\right\}} \mathrm{e}^{-\lambda \tau_{i}^{*}} l\left(\xi_{i}^{*}\right)
\end{aligned}
$$

Taking expectations, we obtain

$$
\begin{aligned}
v(x)-E\left[\mathrm{e}^{-\lambda\left(t \wedge \tau_{n}^{*}\right)} v\left(X_{\left(t \wedge \tau_{n}^{*}\right)+}\right)\right]= & E\left\{\sum _ { i = 1 } ^ { n } \left[\mathbb{1}_{\left\{\tau_{i}^{*} \leq t\right\}} \mathrm{e}^{-\lambda \tau_{i}^{*}} l\left(\xi_{i}^{*}\right)+\int_{t \wedge \tau_{i-1}^{*}}^{t \wedge \tau_{i}^{*}} \mathrm{e}^{-\lambda s} f\left(X_{s}, u_{s}^{*}\right) \mathrm{d} s\right.\right. \\
& \left.\left.-\int_{t \wedge \tau_{i-1}^{*}}^{t \wedge \tau_{i}^{*}} \mathrm{e}^{-\lambda s} v^{\prime}\left(X_{s}\right) \sigma\left(u_{s}^{*}\right) \mathrm{d} B_{s}\right]\right\} .
\end{aligned}
$$

Since the QVI-control is assumed to be admissible, we have $\tau_{n}^{*} \uparrow \infty$. Hence,

$$
\lim _{n \rightarrow \infty} E\left[\mathrm{e}^{-\lambda\left(t \wedge \tau_{n}^{*}\right)} v\left(X_{\left(t \wedge \tau_{n}^{*}\right)+}\right)\right]=E\left[\mathrm{e}^{-\lambda t} v\left(X_{t+}\right)\right] .
$$

We also have

$$
\lim _{n \rightarrow \infty} E\left\{\int_{0}^{t \wedge \tau_{n}^{*}} \mathrm{e}^{-\lambda s} v^{\prime}\left(X_{s}\right) \sigma\left(u_{s}^{*}\right) \mathrm{d} B_{s}\right\}=0 .
$$

Thus, by letting $n \rightarrow \infty$ in (4.1) we get

$$
v(x)-E\left[\mathrm{e}^{-\lambda t} v\left(X_{t+}\right)\right]=E\left\{\sum_{i=1}^{\infty}\left[\mathbb{1}_{\left\{\tau_{i}^{*} \leq t\right\}} \mathrm{e}^{-\lambda \tau_{i}^{*}} l\left(\xi_{i}^{*}\right)+\int_{t \wedge \tau_{i-1}^{*}}^{t \wedge \tau_{i}^{*}} \mathrm{e}^{-\lambda s} f\left(X_{s}, u_{s}^{*}\right) \mathrm{d} s\right]\right\}
$$

It's easy to check that

$$
\lim _{t \rightarrow \infty} E\left[\mathrm{e}^{-\lambda t} v\left(X_{t+}\right)\right]=0
$$

and

$$
\begin{aligned}
& \lim _{t \rightarrow \infty} E\left\{\sum_{i=1}^{\infty}\left[\mathbb{1}_{\left\{\tau_{i}^{*} \leq t\right\}} \mathrm{e}^{-\lambda \tau_{i}^{*}} l\left(\xi_{i}^{*}\right)+\int_{t \wedge \tau_{i-1}^{*}}^{t \wedge \tau_{i}^{*}} \mathrm{e}^{-\lambda s} f\left(X_{s}, u_{s}^{*}\right) \mathrm{d} s\right]\right\} \\
& =E\left\{\sum_{i=1}^{\infty}\left[\mathbb{1}_{\left\{\tau_{i}^{*}<\infty\right\}} \mathrm{e}^{-\lambda \tau_{i}^{*}} l\left(\xi_{i}^{*}\right)+\int_{0}^{\infty} \mathrm{e}^{-\lambda s} f\left(X_{s}, u_{s}^{*}\right) \mathrm{d} s\right]\right\} .
\end{aligned}
$$

Hence, by letting $t \rightarrow \infty$ in (4.2) we have

$$
v(x)=E\left\{\sum_{i=1}^{\infty}\left[\mathbb{1}_{\left\{\tau_{i}^{*}<\infty\right\}} \mathrm{e}^{-\lambda \tau_{i}^{*}} l\left(\xi_{i}^{*}\right)\right]+\int_{0}^{\infty} \mathrm{e}^{-\lambda s} f\left(X_{s}, u_{s}^{*}\right) \mathrm{d} s\right\} .
$$

That is, $v(x)=J^{x}\left(u^{*}, \xi^{*}\right)$. Thus, $\left(u^{*}, \xi^{*}\right)$ constitutes an optimal strategy of the game.

Acknowledgements. The author would like to thank professor Zhen $\mathrm{Wu}$ for helpful discussions and suggestions related to this work. 


\section{REFERENCES}

[1] K.E. Breke and B. Øksendal, A verification theorem for combined stochastic control and impulse control, in Stochastic analysis and related topics VI, J. Decreusefond, J. Gjerde, B. Øksendal and A. Üstünel Eds., Birkhauser, Boston (1997) 211-220.

[2] R. Buckdahn and J. Li, Stochastic differential games and viscosity solutions of Hamiltonian-Jacobi-Bellman-Isaacs equations. SIAM J. Control Optim. 47 (2008) 444-475.

[3] A. Cadenillas and F. Zapatero, Classical and impulse stochastic control of the exchange rate using interest rates and reserves. Math. Finance 10 (2000) 141-156.

[4] M.G. Crandall, H. Ishii and P-L. Lions, User's guide to viscosity solutions of second order partial differential equations. Bull. Amer. Math. Soc. 27 (1992) 1-67.

[5] L.C. Evans and P.E. Souganidis, Differential games and representation formulas for Hamilton-Jacobi equations. Indiana Univ. Math. J. 33 (1984) 773-797.

[6] W.H. Fleming and H.M. Soner, Controlled Markov processes and viscosity solutions. Springer-Verlag, New York (2005).

[7] W.H. Fleming and P.E. Souganidis, On the existence of value functions of two-player, zero-sum stochastic differential games. Indiana Univ. Math. J. 38 (1989) 293-314.

[8] R. Korn, Some applications of impulse control in mathematical finance. Math. Meth. Oper. Res. 50 (1999) $493-518$.

[9] B. Øksendal and A. Sulem, Optimal stochastic impulse control with delayed reaction. Appl. Math. Optim. 58 (2008) $243-255$.

[10] L.C.G. Rogers and D. Williams, Diffusions, Markov processes, and martingales. John Wiley \& Sons, New York (1987).

[11] A.J. Shaiju and S. Dharmatti, Differential games with continuous, switching and impulse controls. Nonlinear Anal. 63 (2005) $23-41$.

[12] J. Yong, Systems governed by ordinary differential equations with continuous, switching and impulse controls. Appl. Math. Optim. 20 (1989) 223-235.

[13] J. Yong, Zero-sum differential games involving impulse controls. Appl. Math. Optim. 29 (1994) 243-261. 